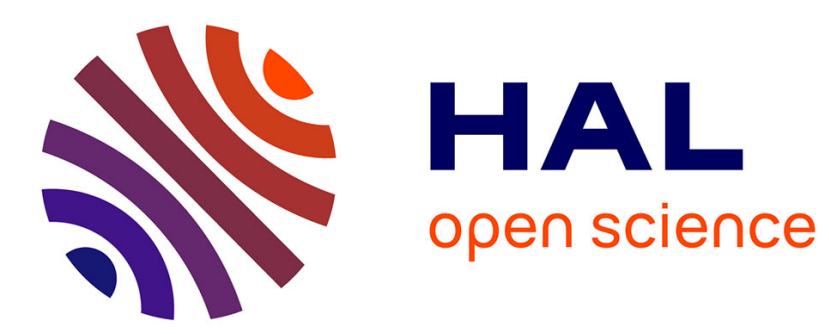

\title{
Doppler broadening in Raman gain spectroscopy at electronic resonance
}

Sylvie A.J. Druet, Jean-Pierre E. Taran, Christian J. Bordé

\section{To cite this version:}

Sylvie A.J. Druet, Jean-Pierre E. Taran, Christian J. Bordé. Doppler broadening in Raman gain spectroscopy at electronic resonance. Journal de Physique, 1980, 41 (2), pp.183-184. 10.1051/jphys:01980004102018300 . jpa-00209232

\section{HAL Id: jpa-00209232 https://hal.science/jpa-00209232}

Submitted on 1 Jan 1980

HAL is a multi-disciplinary open access archive for the deposit and dissemination of scientific research documents, whether they are published or not. The documents may come from teaching and research institutions in France or abroad, or from public or private research centers.
L'archive ouverte pluridisciplinaire HAL, est destinée au dépôt et à la diffusion de documents scientifiques de niveau recherche, publiés ou non, émanant des établissements d'enseignement et de recherche français ou étrangers, des laboratoires publics ou privés. 


\title{
Doppler broadening in Raman gain spectroscopy at electronic resonance $\left({ }^{*}\right)$
}

\author{
S. A. J. Druet and J.-P.-E. Taran \\ ONERA, 92320 Châtillon, France \\ and Ch. J. Bordé \\ Laboratoire de Physique des Lasers, Université Paris-Nord, 93430 Villetaneuse, France
}

(Reçu le 27 août 1979, accepté le $1^{\mathrm{er}}$ octobre 1979)

The recent work of Owyoung has demonstrated the great advantage of stimulated Raman gain spectroscopy (SRGS) to record Raman lines in gases with a high resolution [1]. Because of the interest aroused by this work, especially in view of its possible extension to the domain of electronic resonance enhancement, we feel it necessary to develop the presentation of SRGS at electronic resonance already given in reference [2].

There are several possible experimental approaches to the problem of SRGS : first, one can monitor the gain either at the Stokes or at the pump frequencies ; second, co-or counter-propagating laser beams can be used. This gives a total of 4 distinct instrumental arrangements, among which the one currently in favor is a pump gain/loss measurement using counterpropagating beams [1]. Our discussion of this effect, in connection with figure 14 , was limited to the sole case of Stokes gain measurements using co-propagating laser beams; in addition only 3-level systems were considered. We consider here the four experimental approaches and include the presence of four levels in the molecular system.

If a second excited electronic state $n^{\prime}$ lying under state $n$ is taken into account, four new processes must be added to those already depicted in figure 14 of ref. [2] (we consider only the processes contributing to $\chi^{(3)}\left(-\omega_{2}, \omega_{1},-\omega_{1}, \omega_{2}\right)$ and which do not contain anti-resonances).

The time-ordered diagrams for these processes are shown in figure 1 for the situation of co-propagating laser beams. As an example, figure $2 a$ gives the energymomentum diagram associated with figure $1 c$, and the modification of these two diagrams for counterpropagating waves is presented in figures $2 b$ and $2 c$

\section{(*) ADDENDUM to}

Lineshape and Doppler broadening in resonant CARS and related nonlinear processes through a diagrammatic approach, S. A. J. Druet, J.-P.-E. Taran and Ch. J. Bordé, J. Physique 40 (1979) 819.

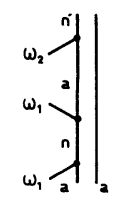

$\omega_{n a}-\omega_{1}-i \gamma_{n a}$

$-i \gamma_{a z}$ $\omega_{n \dot{a}}-\omega_{2}-i \gamma_{n^{\prime} a}$

(a)

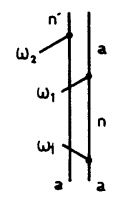

$\omega_{a n}+\omega_{1}-i \gamma_{a n}$

$-i \gamma_{2 a}$ $\omega_{n \dot{a}}-\omega_{2}-i \gamma_{n \dot{a}}$

(b)
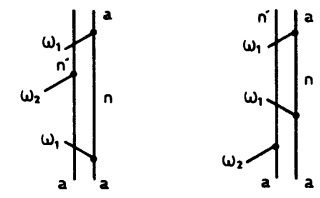

$\omega_{2 n}+\omega_{1}-i \gamma_{a n} \quad \omega_{r_{2}}-\omega_{2}-i \gamma_{n_{2}}$

$\omega_{n+n}+\omega_{1}-\omega_{2}-i \gamma_{n i n} \omega_{n \dot{n}}+\omega_{1}-\omega_{2}-i \gamma_{n i n}$

$\omega_{n^{\prime 2}}-\omega_{2}-i \gamma_{n^{\prime}-a} \quad \omega_{n \dot{a}}-\omega_{2}-i \gamma_{n^{\prime} a}$

(c)

(d)
Fig. 1. - Main diagrams for direct Raman gain spectroscopy on a system with one ground state a and two excited electronic states $n$ and $n^{\prime}$.
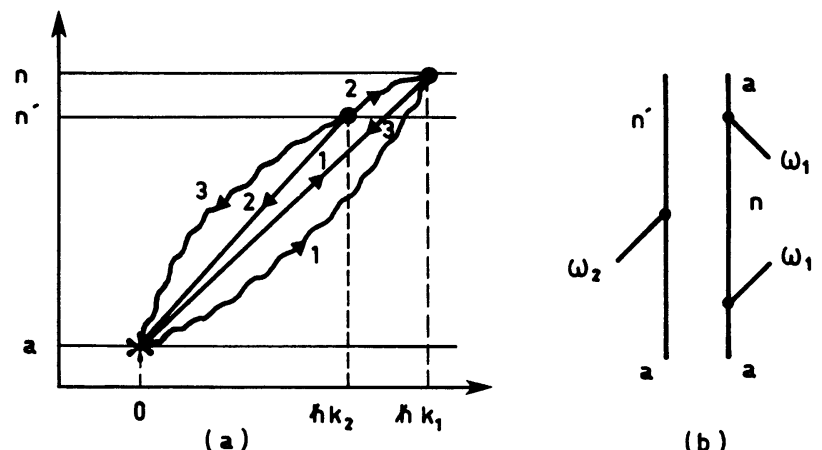

(a)

(b)

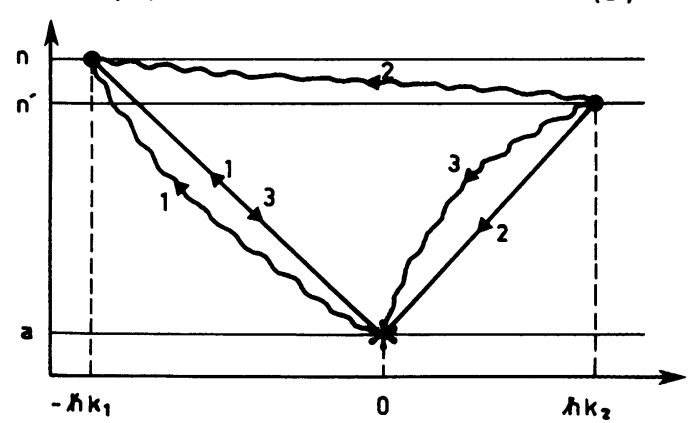

Fig. 2. - (a) Energy-momentum diagram corresponding to figure $1 c ;(b)$ Modification of figure $1 c$ for counter-propagating waves with the direction of $\omega_{1}$, reversed; (c) Corresponding energymomentum diagram.

respectively. If we use the criterion on the wavevector direction for the coherences, as given in [2], it is obvious from figure $2 a$ that the double resonances 
$\left(\omega_{1}=\omega_{n a}, \omega_{2}=\omega_{n a}\right)$ and $\left(\omega_{2}=\omega_{n^{\prime} a}, \omega_{1}-\omega_{2}=\omega_{n n^{\prime}}\right)$ are Doppler-free while $\left(\omega_{1}=\omega_{n a}, \omega_{1}-\omega_{2}=\omega_{n n^{\prime}}\right)$ is not : finally, none of these resonances are Dopplerfree with counter-propagating waves.

For the experimental situation where one monitors the gain at the pump frequency (inverse Raman spectroscopy), one must examine the quantum processes associated with the susceptibility

$$
\chi^{(3)}\left(-\omega_{1}, \omega_{2},-\omega_{2}, \omega_{1}\right) .
$$

Those having a triple resonance are depicted by the time-ordered diagrams of figure 3 for co-propagating waves. Raman gain/loss measurements are thus represented by a total of 16 processes (figs. 14, 1 and 3 ).

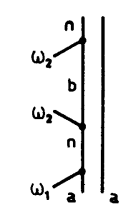

$\omega_{n a}-\omega_{1}-i \gamma_{n a}$ $\omega_{n a}-\omega_{1}-i \gamma_{n a}$

(a)

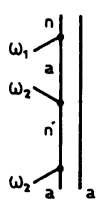

$\omega_{n i a}-\omega_{2}-i \gamma_{n i z}$

$-i \gamma_{\mathbf{a a}}$

$\omega_{n a}-\omega_{1}-i \gamma_{n a}$

(e) $\omega_{b a}-\omega_{1}+\omega_{2}-i \gamma_{b a}$

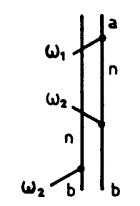

$\omega_{n b}-\omega_{2}-i \gamma_{n b}$

$-i \gamma_{n n}$

$\omega_{n a}-\omega_{1}-i \gamma_{n a}$
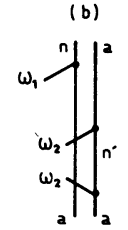

$\omega_{2 n}+\omega_{2}-i \gamma_{a n}$

$-i Y_{a a}$

$\omega_{n a}-\omega_{1}-i \gamma_{n a}$

(f)

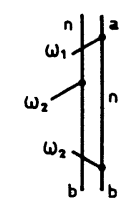

$\omega_{b n}+\omega_{2}-i \gamma_{b n}$

$-i \gamma_{n n}$

$\omega_{n a}-\omega_{1}-i \gamma_{n a}$

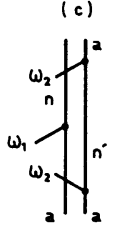

$\omega_{2 n^{\circ}}+\omega_{2}-i \gamma_{a n^{\circ}}$

$\omega_{n n}-\omega_{1}+\omega_{2}-i \gamma_{n n}$

(g)

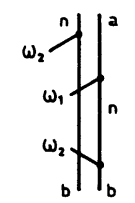

$\omega_{b n}+\omega_{2}-i \gamma_{b n}$

$\omega_{b a}+\omega_{2}-\omega_{1}-i \gamma_{b a}$

$\omega_{n a}-\omega_{1}-i \gamma_{n a}$

(d)

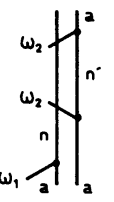

$\omega_{n 2}-\omega_{1}-i \gamma_{n a}$

$\omega_{n a}-\omega_{1}-i \gamma_{n a}$

(h)
Fig. 3. - Main diagrams for inverse Raman scattering.

The results concerning the Doppler character of these processes are summarized in the table. The entry to this table is given according to the following criteria :

1) terms are divided according to the set of chosen levels; energy level diagrams indicate which are the three energy levels coupled by the transitions, out of a total of four levels $a, b, n, n^{\prime}$ under consideration which we choose such that $E_{n}>\mathrm{E}_{n^{\prime}} \gg E_{b}>E_{a}$ (note that the convention on $\mathrm{n}$ and $\mathrm{n}^{\prime}$ is opposite to that used in [2]);

2) the experimental characteristics are subsequently given (direct or inverse Raman measurements, co-or counter-propagating beam arrangement);

3) the susceptibility terms are themselves divided into population [3] (or saturation, or fluorescence) terms, dynamic Stark effect and frequency correlation, (or Raman) terms ;

4) since the modification of the diagrams in going from co-to counter-propagating waves is trivial (see for instance figs. $1 c$ and $2 b$ ), we label the processes for co- and counter-propagating waves by their figure number as they appear in the main manuscript [2] and in this addendum for the co-propagating case.
Table I. - Doppler character of the triple resonances recorded in the Raman gain or inverse Raman gain spectroscopies of four level systems; $1=$ Dopplerfree $; 0=$ Doppler-broadened.

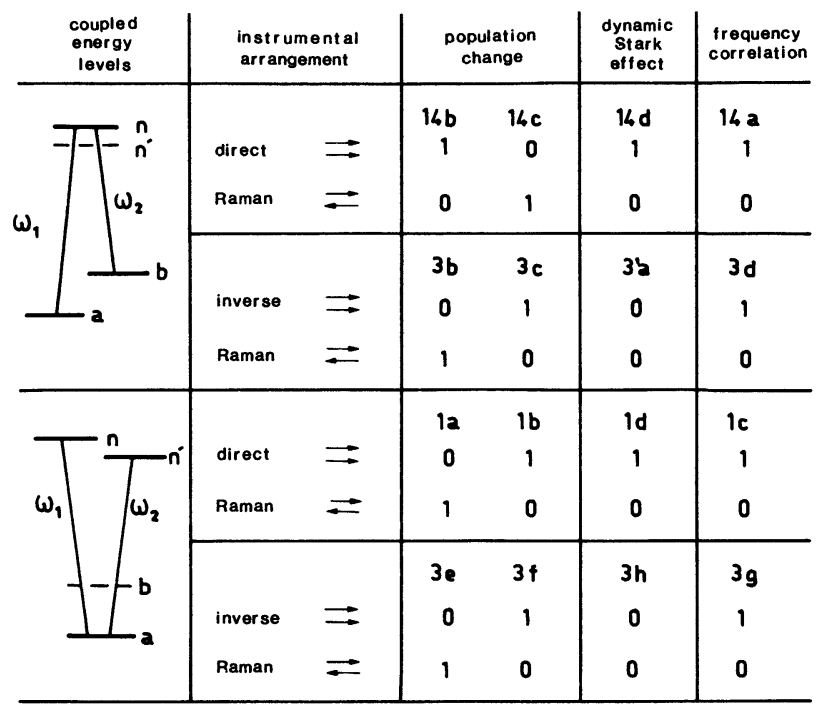

We note that when 2 or more terms give contributions to a given experiment (such as in direct Raman with co-propagating beams), these contributions may add or interfere with one another, leading to distorted lineshapes; a partial discussion of this problem has been conducted by Hänsch and Toschek [3]. For all the contributing diagrams, one can use the general expression for the susceptibility, as given in the Appendix of ref. [2], and which is valid whether on or off resonance.

Two important conclusions can be drawn from this analysis :

- only two Doppler-free terms can be seen in the counter-propagating waves arrangement, whether direct or inverse Raman measurements are made, and these terms are only of the population change (or saturation) type. Thus, Doppler-free Raman resonances cannot be obtained with counter-propagating waves ;

- Raman resonances in the ground or in the excited electronic states can be recorded only with co-propagating beams. Furthermore, only the Raman resonance of the excited electronic state can be seen if $\rho_{b b}^{(0)}=0$ (process $3 \mathrm{~g}$ ) in inverse Raman experiments; if $\rho_{b b}^{(0)} \neq 0$, the $\omega_{a b}$ resonance also appears in the spectrum (process 3d), which is of importance in hot gases or for pure rotational scattering.

\section{References}

[1] Owyoung, A., High resolution coherent Raman spectroscopy of gases (Proceedings 4th International Conference on Laser Spectroscopy, Rottach Egern, June 1979), Eds. Walher, H. and Rothe (Springer Verlag, Berlin) 1979.

[2] Druet, S. A. J., Taran, J.-P.-E. and Bordé, C. J., J. Physique 40 (1979) 819.

[3] Hänsch, Th. and Toschek, P., Z. Phys. 236 (1970) 213. 